\title{
Minimizing earthwork volumes by optimizing vertical alignment with linear programming algorithm
}

\author{
Aytug Artun ${ }^{1}$, Vladimir Badenko' ${ }^{1}$, Yulia Volkova ${ }^{1}$, and Anton Radaev ${ }^{1, *}$ \\ ${ }^{1}$ Peter the Great St.Petersburg Polytechnic University, Politekhnicheskaya str., 29, St.Petersburg, \\ 195251, Russia
}

\begin{abstract}
Earthwork cost is one of the major cost items (approximately $25 \%$ of the construction costs) in road construction projects. The amount of earthwork volumes therefore earthwork cost, mostly depends on the geometry of the road vertical alignment. We can conclude that an optimized vertical alignment has a profound impact on earthwork costs. In this research paper, we solve the task connected to the minimization of earthwork cost by optimizing vertical alignment by the development of linear optimization model with different categories of unknown variables including vertical offsets between road and surface in different points as well as extremum points' numbers. Proposed linear optimization model is supposed to be implemented with application of simplex method as the component of complex branch and bound approach. The developed model has been implemented on the basis of practical example. After the optimization of vertical alignment, earthwork volumes have been calculated in the road design software with average end area volume calculation method. We also have provided numerical results which include earthwork volumes and cost improvements over preliminary design.
\end{abstract}

\section{Introduction}

A road construction project involves many operational steps. Before starting construction, planners need an accurate existing terrain model of the project area in order to design a layout of the road. Road layout (geometry) consists of three main parts: horizontal alignment, vertical alignment (profile), and cross-section. All parts of road geometry should be taken into consideration to design a cost-effective road project. Once the layout of the roadway has been created, general earthwork operations can begin. We will use earthworks term as cut and fill operations in this paper. Cut and fill are the processes of constructing of the roadway which are characterized by large amount of earth material which is being excavated and filled.

Minimizing the earthwork volumes is one of the major tasks of road construction projects. In order to minimize earthwork volumes, one of the most effective ways is to optimize vertical alignment. In this research paper, we will minimize earthwork volumes by optimizing

\footnotetext{
* Corresponding author: radaev_ae@spbstu.ru
} 
vertical alignment with the help of the developed mathematical model in the area of linear programming.

This research paper organized as follows: section 2 presents literature review related with earthwork and vertical alignment optimization; section 3 presents the methodology for the optimization problem; section 4 presents experiment parameters and numerical results; conclusion presents critical analyses of findings, final thoughts, discussions and directions for future works.

Past studies have shown that there were far more vertical alignment optimization models than there were horizontal alignment optimization models and 3D alignment optimization models. The main reason behind this is vertical alignment is easier to design and modify and has influence on fewer cost items (mostly earthwork materials cost) [1-4]. Earthwork cost on a road design includes cost of works such as filling, cutting, and hauling of soil in order to make ground surface ready for the base of the road platform.

We can present existing vertical alignment optimization models in five major categories based on their search algorithms and research models: explicit enumeration [5, 6], numerical search [6, 7], dynamic programming [8-11], genetic algorithms [12-16] and linear programming [17-20].

Table 1 presents weaknesses of the existing vertical road alignment optimization methods.

Table 1. Weaknesses of the existing vertical road alignment optimization methods.

\begin{tabular}{|c|c|}
\hline Type of Model & Weaknesses and Deficiencies \\
\hline Explicit Enumeration & $\begin{array}{l}\text { - Inefficient, cumbersome } \\
\text { - } \quad \text { Not suitable for continuous search space }\end{array}$ \\
\hline Numerical Search & $\begin{array}{ll}\text { - } & \text { Very complex modelling } \\
\text { - } & \text { Difficulty in handling discontinuous cost factors } \\
\text { - } & \text { Tendency to get trapped in local optima } \\
\end{array}$ \\
\hline Dynamic Programming & $\begin{array}{ll}\text { - } & \text { Outputs are not smooth } \\
\text { - } & \text { Not applicable for implicit functions } \\
\text { - } & \text { Not suitable for continuous search space } \\
\text { - } & \text { Requires independencies among subproblems }\end{array}$ \\
\hline Genetic Algorithm & $\begin{array}{l}\text { - } \quad \text { Outputs are not smooth } \\
\text { - } \quad \text { Complex modelling }\end{array}$ \\
\hline Linear Programming & $\begin{array}{l}\text { - } \\
\text { - } \quad \text { Only covers limited points for constraints } \\
\end{array}$ \\
\hline
\end{tabular}

On the light of this observations, it is safe to say that all those optimization models have some critical weaknesses and deficiencies when applied to the road alignments in real-world scenarios.

\section{Methods}

The model has been developed in order to minimize earthwork costs (cut and fill volumes) by optimizing vertical alignment elevations. In order to solve vertical alignment optimization problem, we supposed using methods of numerical analysis, namely simplex method in combination with branch and bound approach for processing of intermediate results. Corresponding procedure supposes iterative modification of the initial model's structure by selecting integer variable (which current value is not integer) and forming of two alternative variants of model's structure with additional direct constraints [17].

During the optimization process, the calculation of cut and fill volumes is approximated and based on only the elevation difference between the existing ground surface elevations 
and the vertical alignment design elevations along the stations of the horizontal alignment. Only after the optimization process, accurate earthwork volumes will be calculated with the Average End Area and the Prismoidal Method in AutoCAD Civil 3D software.

The developed linear optimization model provides integrated solution which includes optimization of vertical alignment (with earthwork cost minimization in mind), and accurate calculation of earthwork volumes (which is based on the terrain models extracted from GIS databases and created with GIS software tools) with the help of road design software.

Table 2 presents initial data, Table 3 presents unknown variables, and Table 4 presents calculated characteristics for the developed optimization model. Profiles are divided into sections and are given point numbers with 20 -meter sampling rate. A section on a station is called fill (respectively cut), if the elevation difference between the design profile of the road and the existing ground profile is positive (respectively negative). In reality, existing ground and road elevations vary in a cross-section, so this optimization works with approximate volumes which is based on elevation differences only along the centerline.

Table 2. Initial data for the developed optimization model.

\begin{tabular}{|l|l|c|l|}
\hline No. & Parameter name & $\begin{array}{l}\text { Meas. } \\
\text { units }\end{array}$ & Sign / expression \\
\hline 1 & Generalized parameters of task & \multicolumn{2}{|l|}{} \\
\hline 1.1 & Cost factor for embankment & $\mathrm{CU} / \mathrm{m}$ & $c^{+}$ \\
\hline 1.2 & Cost factor for cut & $\mathrm{CU} / \mathrm{m}$ & $c^{-}$ \\
\hline 1.3 & Elementary vertical offset & $\mathrm{m}$ & $\Theta$ \\
\hline 1.4 & Constraint constant & - & $\mathrm{M}$ \\
\hline 1.5 & $\begin{array}{l}\text { Maximal absolute positive } \\
\text { offset }\end{array}$ & $\mathrm{m}$ & $\Delta^{+\max }$ \\
\hline 1.6 & $\begin{array}{l}\text { Maximal absolute negative } \\
\text { offset }\end{array}$ & $\mathrm{m}$ & $\Delta^{-\max }$ \\
\hline
\end{tabular}

Table 2. Initial data for the developed optimization model (continued).

\begin{tabular}{|l|l|c|l|}
\hline No. & Parameter name & $\begin{array}{l}\text { Meas. } \\
\text { units }\end{array}$ & Sign / expression \\
\hline 1.7 & $\begin{array}{l}\text { Maximal absolute difference } \\
\text { between offsets in transitions }\end{array}$ & $\mathrm{m}$ & $\sigma$ \\
\hline 1.8 & $\begin{array}{l}\text { Maximal absolute difference } \\
\text { between embankments and } \\
\text { cuts }\end{array}$ & $\mathrm{m}$ & $\varepsilon$ \\
\hline 2 & Generalized parameters of profile & \multicolumn{1}{|l|}{} \\
\hline 2.1 & Points number & units & $m$ \\
\hline 2.2 & Convexity intervals number & units & $n^{\text {conv }}=\sum_{i=1}^{m} \phi_{i}^{-+}$ \\
\hline 2.3 & Concavity intervals number & units & $n^{\text {conc }}=\sum_{i=1}^{m} \phi_{i}^{+-}$ \\
\hline 2.4 & $\begin{array}{l}\text { Generalized transitions } \\
\text { number }\end{array}$ & units & $n^{\text {tr }}=\sum_{i=1}^{m} \phi_{i}^{0}$ \\
\hline 3 & Indexes & - & $i$ \\
\hline 3.1 & Index for points & - & $k^{\prime}$ \\
\hline 3.2 & Index for convexity intervals &
\end{tabular}




\begin{tabular}{|l|l|c|l|l|}
\hline 3.3 & Index for concavity intervals & - & $k^{\prime \prime}$ \\
\hline 3.4 & $\begin{array}{l}\text { Index for generalized } \\
\text { transitions }\end{array}$ & - & $k^{\prime \prime \prime}$ \\
\hline 4 & Parameters for each point $i, i=1,2, \ldots, m$ \\
\hline 4.1 & Surface coordinate & $\mathrm{m}$ & $z_{i}^{0}$ \\
\hline 4.2 & $\begin{array}{l}\text { Convexity / concavity } \\
\text { indicator }\end{array}$ & - & $\varphi_{i}$ \\
\hline 4.3 & Road minimal elevation & $\mathrm{m}$ & $z_{i}^{\text {min }}$ \\
\hline 4.4 & Road maximal elevation & $\mathrm{m}$ & $z_{i}^{\max }$ \\
\hline 4.5 & $\begin{array}{l}\text { Concavity-convexity } \\
\text { transition indicator }\end{array}$ & - & $\phi_{i}^{-+}=\left\{\begin{array}{l}\operatorname{sign}\left(\varphi_{i}\right), \text { if } i>1 \wedge \\
1, i>\varphi_{i}=1, \varphi_{i-1}=0 \\
0, \text { otherwise } .\end{array}\right.$ \\
\hline 4.6 & $\begin{array}{l}\text { Convexity-concavity } \\
\text { transition indicator }\end{array}$ & - & $\phi_{i}^{+-}=\left\{\begin{array}{l}1-\operatorname{sign}\left(\varphi_{i}\right), \text { if } i=1 ; \\
1, i>1 \wedge \varphi_{i}=0, \varphi_{i-1}=1 ; \\
0, \text { otherwise } .\end{array}\right.$ \\
\hline 4.7 & $\begin{array}{l}\text { Generalized transition } \\
\text { indicator**** }\end{array}$ & - & $\phi_{i}^{0}=\phi_{i}^{-+}+\phi_{i}^{+-}$ \\
\hline
\end{tabular}

Table 2. Initial data for the developed optimization model (continued).

\begin{tabular}{|c|c|c|c|}
\hline No. & Parameter name & \begin{tabular}{|l|} 
Meas. \\
units
\end{tabular} & Sign / expression \\
\hline 4.8 & $\begin{array}{l}\text { Cumulate concavity-convexity } \\
\text { transition indicator }\end{array}$ & - & $\phi_{i}^{-+\Sigma}=\sum_{i^{\prime}=1}^{i} \phi_{i^{\prime}}^{-+}$ \\
\hline 4.9 & $\begin{array}{l}\text { Cumulate convexity-concavity } \\
\text { transition indicator }\end{array}$ & - & $\phi_{i}^{+-\Sigma}=\sum_{i^{\prime}=1}^{i} \phi_{i^{\prime}}^{+-}$ \\
\hline 4.10 & $\begin{array}{l}\text { Cumulate generalized } \\
\text { transition indicator }\end{array}$ & - & $\phi_{i}^{0 \Sigma}=\sum_{i^{\prime}=1}^{i} \phi_{i^{\prime}}^{0}$ \\
\hline 5 & \multicolumn{3}{|c|}{ Parameters for each convexity interval $k^{\prime}, k^{\prime}=1,2, \ldots, n^{\text {conv }}$} \\
\hline 5.1 & Start point number & - & $i_{k^{\prime}}^{\text {conv min }}=\min \left\{i \mid \phi_{i}^{-+\Sigma}=k^{\prime}\right\}$ \\
\hline 5.2 & End point number & - & $\begin{array}{l}i_{k^{\prime}}^{\text {conv max }}= \\
=\left\{\begin{array}{l}\min \left\{i \mid \phi_{i}^{+-\Sigma}=k^{\prime}+1-\operatorname{sign}\left(\varphi_{i=1}\right)\right\}-1, \\
\text { if } k^{\prime}+1-\operatorname{sign}\left(\varphi_{i=1}\right) \leq n^{\text {conc }} ; \\
m, \text { otherwise. }\end{array}\right.\end{array}$ \\
\hline 6 & \multicolumn{3}{|c|}{ Parameters for each concavity interval $k^{\prime \prime}, k^{\prime \prime}=1,2, \ldots, n^{\text {conc }}$} \\
\hline 6.1 & Start point number & - & $i_{k^{\prime}}^{\text {conc min }}=\min \left\{i \mid \phi_{i}^{+-\Sigma}=k^{\prime}\right\}$ \\
\hline 6.2 & End point number & - & $\begin{array}{l}i_{k^{\prime}}^{\text {conc max }}= \\
=\left\{\begin{array}{l}\min \left\{i \mid \phi_{i}^{-+\Sigma}=k^{\prime}+1-\operatorname{sign}\left(\varphi_{i=1}\right)\right\}-1, \\
\quad \text { if } k^{\prime}+1-\operatorname{sign}\left(\varphi_{i=1}\right) \leq n^{\text {conv }} ; \\
m, \text { otherwise. }\end{array}\right.\end{array}$ \\
\hline
\end{tabular}




\begin{tabular}{|l|l|c|l|}
\hline 7 & \multicolumn{3}{|l|}{ Parameters for each generalized transition $k^{\prime \prime \prime}, k^{\prime \prime \prime}=1,2, \ldots, n^{\text {tr }}$} \\
\hline 7.1 & Start point number & - & $i_{k^{\prime}}^{\text {tr min }}=\max \left\{i \mid \phi_{i}^{0 \Sigma}=k^{\prime \prime \prime}-1\right\}$ \\
\hline 7.2 & End point number & - & $i_{k^{\prime}}^{\text {tr max }}=\min \left\{i \mid \phi_{i}^{0 \Sigma}=k^{\prime \prime \prime}\right\}$ \\
\hline
\end{tabular}

Notes:

* $\quad$ arbitrary real positive number; $M>>\max \left(m, \max \left\{z_{i}^{0}\right\}\right)$;

** convexity / concavity indicator for point $i$ is equal to 1 if the point belongs to convexity interval, otherwise indicator equals 0 ;

*** concavity-convexity transition indicator for point $i$ is equal to 1 if there is increasing of convexity / concavity indicator from the value 0 in previous point $(i-1)$ to the value 1 in current point $(i)$; otherwise the indicator equals 0 ;

***** convexity-concavity transition indicator for point $i$ is equal to 1 if there is decreasing of convexity / concavity indicator from the value 1 in previous point $(i-1)$ to the value 0 in current point $(i)$; otherwise the indicator equals 0 ;

****** generalized transition indicator for point $i$ is equal to 1 if values of convexity / concavity indicator in previous $(i-1)$ and current $(i)$ points are not equal; otherwise the indicator equals 0 .

Table 3. Unknown variables of the developed optimization model.

\begin{tabular}{|c|c|c|c|}
\hline No. & Variable name & $\begin{array}{l}\text { Meas. } \\
\text { units }\end{array}$ & Sign \\
\hline 1 & \multicolumn{3}{|c|}{ Variables for each point $i, i=1,2, \ldots, m$} \\
\hline 1.1 & Positive offset ${ }^{*}$ & $\mathrm{~m}$ & $\Delta_{i}^{+}$ \\
\hline 1.2 & Negative offset ${ }^{*}$ & $\mathrm{~m}$ & $\Delta_{i}^{-}$ \\
\hline 1.3 & Embankment / cut indicator ${ }^{* *}$ & - & $e_{i}$ \\
\hline 1.4 & $\begin{array}{l}\text { Road coordinate increase / decrease } \\
\text { indicator }\end{array}$ & - & $y_{i}$ \\
\hline 2 & \multicolumn{3}{|c|}{ Variables for each convexity interval $k^{\prime}, k^{\prime}=1,2, \ldots, n^{\text {conv }}$} \\
\hline 2.1 & Embankment extremum point number & - & $i_{k^{\prime}}^{\text {conv }}$ \\
\hline 3 & \multicolumn{3}{|c|}{ Variables for each concavity interval $k^{\prime \prime}, k^{\prime \prime}=1,2, \ldots, n^{\text {conc }}$} \\
\hline 3.1 & Cut extremum point number & - & $i_{k^{\prime \prime}}^{\text {conc }}$ \\
\hline
\end{tabular}

Notes:

* variable has double (non-negative) value;

** embankment / cut indicator for point $\mathrm{i}$ is equal to 1 if road coordinate is bigger than surface coordinate (i.e. embankment is required) in considered point; otherwise the indicator equals 0 ; *** road coordinate increase / decrease indicator for point $i$ is equal to 1 , if road coordinate in current point $(i)$ is bigger or equal to the corresponding value in previous point $(i-1)$; otherwise the indicator equals 0 .

Table 4. Calculated characteristics of the developed optimization model.

\begin{tabular}{|l|l|c|l|}
\hline No. & Calculated characteristic name & $\begin{array}{l}\text { Meas. } \\
\text { units }\end{array}$ & Formula \\
\hline 1 & \multicolumn{1}{|l|}{ Calculated characteristics for each point $i, i=1,2, \ldots, m$} \\
\hline 1.1 & Road elevation & $\mathrm{m}$ & $z_{i}\left(\Delta_{i}^{+}, \Delta_{i}^{-}\right)=z_{i}^{0}+\Delta_{i}^{+}-\Delta_{i}^{-}$ \\
\hline 1.2 & $\begin{array}{l}\text { Difference of road coordinate's } \\
\text { values in current }(i) \text { and previous } \\
(i-1) \text { points }\end{array}$ & $\mathrm{m}$ & $\left.\begin{array}{l}\Delta_{i}\left(\Delta_{i}^{+}, \Delta_{i}^{-}, \Delta_{i-1}^{+}, \Delta_{i-1}^{-}\right)= \\
=z_{i}\left(\Delta_{i}^{+}, \Delta_{i}^{-}\right)-z_{i-1}\left(\Delta_{i-1}^{+}, \Delta_{i-1}^{-}\right.\end{array}\right)$ \\
\hline 2 & Generalized calculated characteristics for the profile \\
\hline 2.1 & Summarized costs & $\mathrm{CU}$ & $C^{\Sigma}\left(\left\{\Delta_{i}^{+}\right\},\left\{\Delta_{i}^{-}\right\}\right)=c^{+} \cdot \sum_{i=1}^{m} \Delta_{i}^{+}+c^{-} \cdot \sum_{i=1}^{m} \Delta_{i}^{-}$ \\
\hline
\end{tabular}




\section{Results and discussion}

The structure of the developed optimization model is described by the expressions (1) and (2). The objective function of vertical alignment optimization problem is the total earthworks cost. Direct constraints for the unknown variables describe the lower and upper bounds for corresponding values as well as connection of the variables' values with binary or integer set. Functional constraints determine connection between different categories of unknown variables to make optimal vertical alignment trajectory smooth.

$$
\begin{aligned}
& C^{\Sigma}\left(\left\{\Delta_{i}^{+}\right\},\left\{\Delta_{i}^{-}\right\}\right) \rightarrow \min \\
& 0 \leq \Delta_{i}^{+} \leq \Delta_{i}^{+\max }, i=1,2, \ldots, m \text {; } \\
& 0 \leq \Delta_{i}^{-} \leq \Delta_{i}^{\text {-mx }}, i=1,2, \ldots, m \text {; } \\
& e_{i} \in\{0 ; 1\}, i=1,2, \ldots, m \text {; } \\
& y_{i} \in\{0 ; 1\}, i=1,2, \ldots, m \text {; } \\
& i_{k^{\prime}}^{\text {conv min }} \leq i_{k^{\prime}}^{\text {conv }} \leq i_{k^{\prime}}^{\text {conv max }}, k^{\prime}=1,2, \ldots, n^{\text {conv }} ; \\
& i_{k^{\prime \prime}}^{\text {conc min }} \leq i_{k^{\prime \prime}}^{\text {conc }} \leq i_{k^{\prime \prime}}^{\text {conc max }}, k^{\prime \prime}=1,2, \ldots, n^{\text {conc }} \text {; } \\
& i_{k^{\prime}}^{\text {conv }} \in Z, k^{\prime}=1,2, \ldots, n^{\text {conv }} \\
& i_{k^{\prime \prime}}^{\text {conc }} \in Z, k^{\prime \prime}=1,2, \ldots, n^{\text {conc }} \text {; } \\
& \Delta_{i}^{+} \leq \mathrm{M} \cdot e_{i}, i=1,2, \ldots, m ; \\
& \Delta_{i}^{-} \leq \mathrm{M} \cdot\left(1-e_{i}\right), i=1,2, \ldots, m \text {; } \\
& \frac{i_{k^{\prime}}^{\text {conv }}}{i}-y_{i} \geq 0, k^{\prime}=1,2, \ldots, n^{\text {conv }} ; i_{k^{\prime}}^{\text {conv min }} \leq i \leq i_{k^{\prime}}^{\text {conv max }} ; \varphi_{i}=1 \text {; } \\
& i \in\{1 ; 2 ; \ldots ; m\} \text {; } \\
& \left\{\frac{i_{k^{\prime}}^{\text {conv }}}{i}-1-\mathrm{M} \cdot y_{i} \leq 0, k^{\prime}=1,2, \ldots, n^{\text {conv }} ; i_{k^{\prime}}^{\text {conv nin }} \leq i \leq i_{k^{\prime}}^{\text {conv max }} ; \varphi_{i}=1 ;\right. \\
& i \in\{1 ; 2 ; \ldots ; m\} \text {; } \\
& \frac{i_{k^{\prime}}^{\text {conc }}}{i}+y_{i}-1 \geq 0, k^{\prime \prime}=1,2, \ldots, n^{\text {conv }} ; i_{k^{\prime \prime}}^{\text {conn nin }} \leq i \leq i_{k^{\prime \prime}}^{\text {conc max }} ; \varphi_{i}=0 \\
& i \in\{1 ; 2 ; \ldots ; m\} \text {; } \\
& \frac{i_{k^{\prime \prime}}^{\text {conc }}}{i}-1-\mathrm{M} \cdot\left(1-y_{i}\right) \leq 0, k^{\prime \prime}=1,2, \ldots, n^{\text {conv }} ; i_{k^{\prime \prime}}^{\text {conn nin }} \leq i \leq i_{k^{\prime}}^{\text {conc max }} ; \varphi_{i}=0 \text {; } \\
& \Delta_{i}\left(\Delta_{i}^{+}, \Delta_{i}^{-}, \Delta_{i-1}^{+}, \Delta_{i-1}^{-}\right) \leq \sigma, i=2,3, \ldots, m ; \\
& i \in\{1 ; 2 ; \ldots ; m\} \text {; } \\
& \Delta_{i}\left(\Delta_{i}^{+}, \Delta_{i}^{-}, \Delta_{i-1}^{+}, \Delta_{i-1}^{-}\right) \geq-\sigma, i=2,3, \ldots, m \text {; } \\
& \Delta_{i}\left(\Delta_{i}^{+}, \Delta_{i}^{-}, \Delta_{i-1}^{+}, \Delta_{i-1}^{-}\right) \leq \mathrm{M} \cdot y_{i}, i=2,3, \ldots, m ; \\
& \Delta_{i}\left(\Delta_{i}^{+}, \Delta_{i}^{-}, \Delta_{i-1}^{+}, \Delta_{i-1}^{-}\right) \geq-\mathrm{M} \cdot\left(1-y_{i}\right), i=2,3, \ldots, m ; \\
& \Delta_{i}\left(\Delta_{i}^{+}, \Delta_{i}^{-}, \Delta_{i-1}^{+}, \Delta_{i-1}^{-}\right) \geq\left(i_{k^{\prime}}^{\text {conv }}-i\right) \cdot \Theta-\mathrm{M} \cdot y_{i}, k^{\prime}=1,2, \ldots, n^{\text {conv }} ; \\
& i_{k^{\prime}}^{\text {conv nin }} \leq i \leq i_{k^{\prime}}^{\text {conv max }} ; \varphi_{i}=1 ; i \in\{2 ; 3 ; \ldots ; m\} \text {; } \\
& \Delta_{i}\left(\Delta_{i}^{+}, \Delta_{i}^{-}, \Delta_{i-1}^{+}, \Delta_{i-1}^{-}\right) \leq\left(i_{k^{\prime}}^{\text {conv }}-i\right) \cdot \Theta+\mathrm{M} \cdot\left(1-y_{i}\right), k^{\prime}=1,2, \ldots, n^{\text {conv }} ; \\
& i_{k^{\prime}}^{\text {conv nin }} \leq i \leq i_{k^{\prime}}^{\text {conv max }} ; \varphi_{i}=1 ; i \in\{2 ; 3 ; \ldots ; m\}
\end{aligned}
$$




$$
\begin{aligned}
& \Delta_{i}\left(\Delta_{i}^{+}, \Delta_{i}^{-}, \Delta_{i-1}^{+}, \Delta_{i-1}^{-}\right) \geq\left(i-i_{k^{\prime}}^{\mathrm{conc}}\right) \cdot \Theta-\mathrm{M} \cdot y_{i}, k^{\prime \prime}=1,2, \ldots, n^{\mathrm{conc}} ; \\
& i_{k^{\prime \prime}}^{\text {conc min }} \leq i \leq i_{k^{\prime \prime}}^{\text {conc max }} ; \varphi_{i}=1 ; i \in\{2 ; 3 ; \ldots ; m\} ; \\
& \Delta_{i}\left(\Delta_{i}^{+}, \Delta_{i}^{-}, \Delta_{i-1}^{+}, \Delta_{i-1}^{-}\right) \leq\left(i-i_{k^{\prime \prime}}^{\text {conc }}\right) \cdot \Theta+\mathrm{M} \cdot\left(1-y_{i}\right), k^{\prime \prime}=1,2, \ldots, n^{\text {conc }} ; \\
& i_{k^{\prime \prime}}^{\text {conc min }} \leq i \leq i_{k^{\prime \prime}}^{\text {conc max }} ; \varphi_{i}=1 ; i \in\{2 ; 3 ; \ldots ; m\} ; \\
& \Delta_{i}\left(\Delta_{i}^{+}, \Delta_{i}^{-}, \Delta_{i-1}^{+}, \Delta_{i-1}^{-}\right)-\Delta_{i-1}\left(\Delta_{i-1}^{+}, \Delta_{i-1}^{-}, \Delta_{i-2}^{+}, \Delta_{i-2}^{-}\right) \leq 0, \varphi_{i}=1 ; i \in\{3 ; 4 ; \ldots ; m\} ; \\
& \left\{\Delta_{i}\left(\Delta_{i}^{+}, \Delta_{i}^{-}, \Delta_{i-1}^{+}, \Delta_{i-1}^{-}\right)-\Delta_{i-1}\left(\Delta_{i-1}^{+}, \Delta_{i-1}^{-}, \Delta_{i-2}^{+}, \Delta_{i-2}^{-}\right) \geq 0, \varphi_{i}=0 ; i \in\{3 ; 4 ; \ldots ; m\} ;\right. \\
& z_{i}\left(\Delta_{i}^{+}, \Delta_{i}^{-}\right) \geq z_{i}^{\text {nin }}, i=1,2, \ldots, m \\
& z_{i}\left(\Delta_{i}^{+}, \Delta_{i}^{-}\right) \leq z_{i}^{\operatorname{mx}}, i=1,2, \ldots, m ;
\end{aligned}
$$

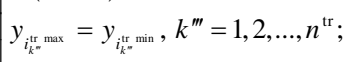

$$
\begin{aligned}
& \sum_{i=1}^{m} \Delta_{i}^{+}-\sum_{i=1}^{m} \Delta_{i}^{-} \leq \varepsilon \\
& \sum_{i=1}^{m} \Delta_{i}^{+}-\sum_{i=1}^{m} \Delta_{i}^{-} \geq-\varepsilon,
\end{aligned}
$$

where $Z-$ set of integer values; $Z=\{\ldots ;-1 ; 0 ; 1 ; \ldots\}$.

Previously described parameters, variables, and constraints are formulated and used in MS Excel software. Once all matrices and tables are created, all data is imported to MATLAB software to solve vertical alignment optimization problem.

The developed model was implemented on the practical example. Study area is located at the east of Ufa City near Aratskoye village in Chelyabinsk oblast of Russia. We have created and fixed horizontal alignment for the vertical alignment optimization problem. Created horizontal alignment is $3627.522 \mathrm{~m}$, which yields total 183 station points along the alignment with $20 \mathrm{~m}$ sampling rate. We will visualize and compare existing ground elevations and experimental vertical alignment profiles with these station-elevation points.

Fig. 1, $a$ represents the key map of the study area. Fig. 1, $b$ represents horizontal alignment and its parameters which are created in the AutoCAD Civil 3D software package for the vertical alignment optimization problem.

a)

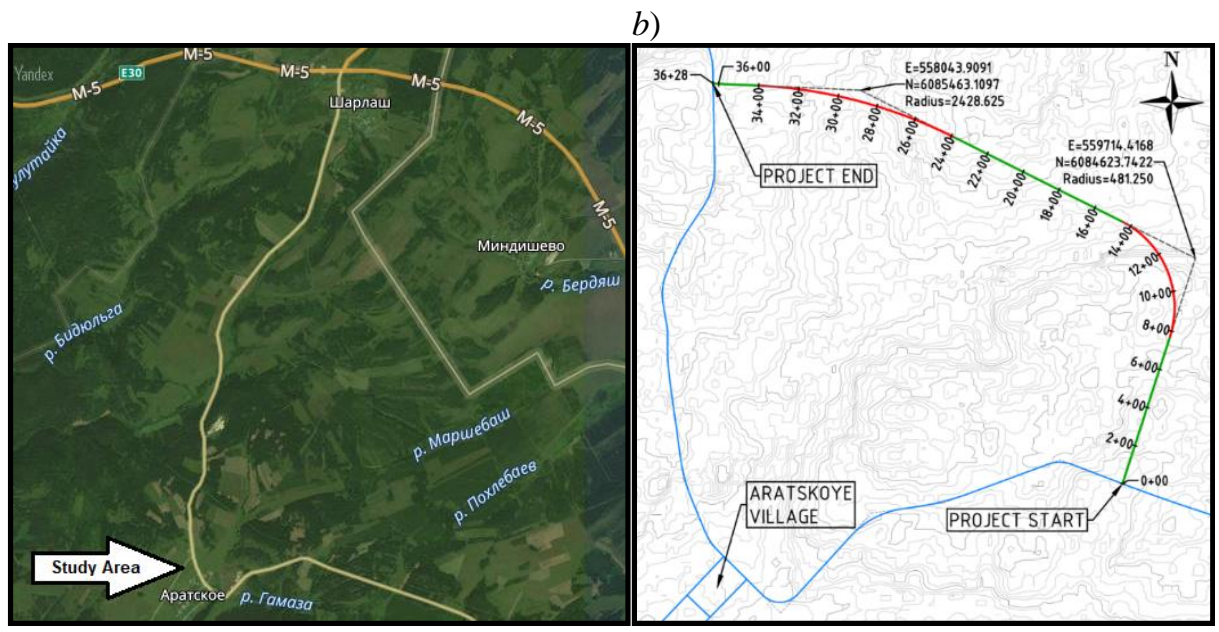

Fig. 1. Study area key map acquired from Yandex $(a)$ and study area horizontal alignment $(3627.5 \mathrm{~m})$ created and fixed in AutoCAD Civil 3D software for the vertical alignment optimization $(b)$

Table 5 presents vertical alignment optimization parameters which are used through experiments. Corresponding results are shown in Fig. 2. 
Table 5. Vertical alignment optimization experiment parameters.

\begin{tabular}{|l|c|c|c|c|c|}
\hline Optimization Experiment No & Sign & $\begin{array}{c}\text { Meas. } \\
\text { unit }\end{array}$ & $\mathbf{1}$ & $\mathbf{2}$ & $\mathbf{3}$ \\
\hline Points number & $m$ & units & 183 & 183 & 183 \\
\hline Convexity intervals number & $n^{\text {conv }}$ & units & 3 & 2 & 3 \\
\hline Concavity intervals number & $n^{\text {conc }}$ & units & 2 & 3 & 3 \\
\hline Generalized transitions number & $n^{\text {tr }}$ & units & 4 & 4 & 5 \\
\hline Cost factor for fill & $c^{+}$ & $\mathrm{CU} / \mathrm{m}$ & 10 & 10 & 4 \\
\hline Cost factor for cut & $c^{-}$ & $\mathrm{CU} / \mathrm{m}$ & 3 & 3 & 10 \\
\hline Elementary vertical offset & $\Theta$ & $\mathrm{m}$ & 0.125 & 0.075 & 0.1 \\
\hline Constraint constant & $\mathrm{M}$ & - & 10000 & 10000 & 10000 \\
\hline Maximal absolute positive offset & $\Delta^{+\max }$ & $\mathrm{m}$ & 25 & 40 & 22 \\
\hline Maximal absolute negative offset & $\Delta^{\text {max }}$ & $\mathrm{m}$ & 25 & 40 & 22 \\
\hline $\begin{array}{l}\text { Maximal absolute difference } \\
\text { between offsets in transitions }\end{array}$ & $\sigma$ & $\mathrm{m}$ & 2 & 3 & 2 \\
\hline $\begin{array}{l}\text { Maximal absolute difference } \\
\text { between fills and cuts }\end{array}$ & $\varepsilon$ & $\mathrm{m}$ & 100 & 1000 & 100 \\
\hline Road elevation upper limit & $z_{i}^{\text {min }}$ & $\mathrm{m}$ & 481.054 & 480.000 & 480.000 \\
\hline Road elevation lower limit & $z_{i}^{\text {max }}$ & $\mathrm{m}$ & 433.255 & 430.000 & 430.000 \\
\hline
\end{tabular}

a)

b)

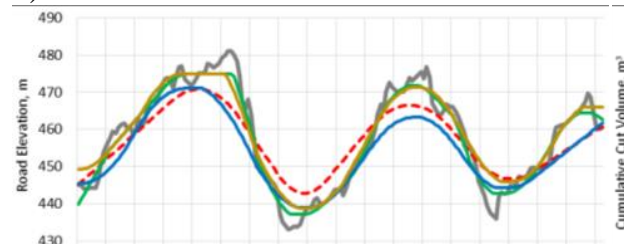

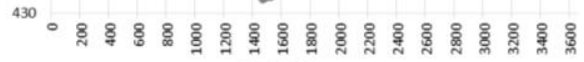
Road Station, $m$

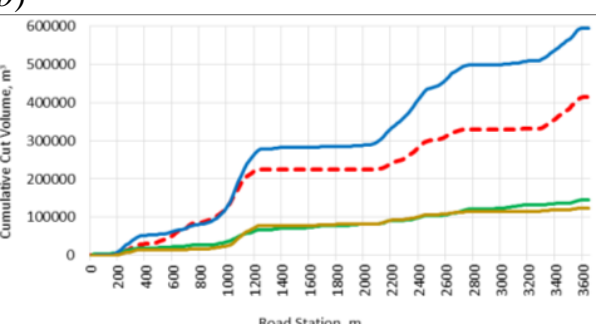

c)
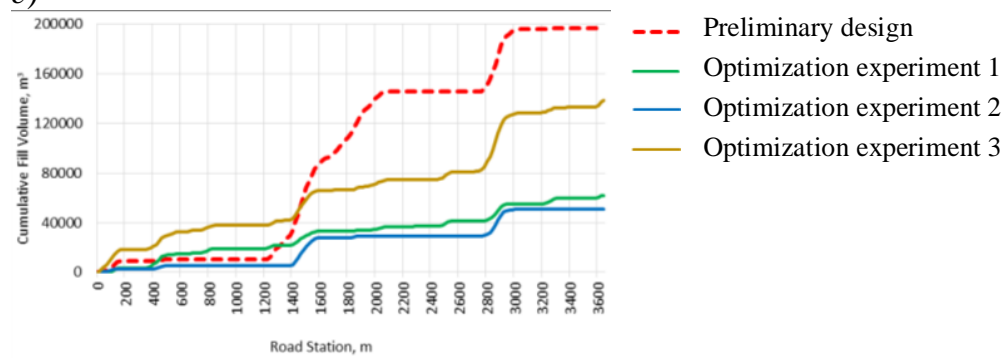

Fig. 2. Comparison of vertical alignment or profile (a), cumulative cut (b) and cumulative fill volume (c) optimization over preliminary design

Fig. 2,a is graphical representation of optimization profiles. The effects of cost factor and elementary vertical offset parameters are clearly shown. In Experiment 1, algorithm prefers cuts over fills (with 10 fill and 3 cut cost factor) and experiment 1 profile has sharper transitions with the biggest elementary offset value $(0.125)$. On the other hand, smaller elementary vertical offset value (0.075) used in experiment 2 makes profile considerably smoother, therefore less intense. In Experiment 3 with different cost factors (4 fill cost factor, 
10 cut cost factor), algorithm prefers fills over cuts and with middle elementary offset value (0.1), transitions are in between experiment 1 and experiment 2.

After the optimization of vertical alignments, we have done accurate earthworks volume calculations based on cross-sectional areas with the help of AutoCAD Civil 3D software. Volume calculations depict the differences between the experiments (therefore cost factor for fill-cut and elementary vertical offset parameters) more clearly.

Fig. 2,b presents comparison of cumulative cut volumes between optimization experiments and preliminary design along stations. Cumulative cut volumes of optimization experiment 1 and 3 have been improved over preliminary design. However, cumulative cut volumes of optimization experiment 2 have been deteriorated compared to preliminary design. The main reason behind this is that elementary vertical offset value (0.075) used in experiment 2 is so strict and doesn't allow algorithm to use sharp transitions. Consequently, this results in without any cut volume improvements especially with selected fill-cut cost factors (10 for fill and 3 for cut).

Fig. 2,c presents comparison of cumulative fill volumes between optimization experiments and preliminary design along stations. As shown in the figure, cumulative fill volumes of all experiments have been improved over preliminary design. However, improvements on experiment 1 and 2 are considerably higher than those on experiment 3 . The main reason behind this is that fill-cut cost factors used in experiment 1 and 2 (10 for fill and 3 for cut) makes algorithm prefer fill volume improvement over cut volume improvement.

In order to evaluate the optimization experiments better, we have calculated hypothetical earthworks cost. Unit costs for fill and cut have been converted from cost factors given in table 5 for each experiment. It was concluded that optimization experiment 1, 2 and 3 provides $67 \%, 28 \%$, and $68 \%$ total earthwork cost reduction respectively.

\section{Conclusions}

Minimizing earthwork volumes by optimizing vertical alignment is a difficult road engineering problem for computers to solve without human guidance. Computerized methods are crucial to automate the optimization to determine the solution that incurs the minimal earthwork costs. In this paper, we developed a linear optimization model which ensures optimization of vertical alignment over a fixed horizontal alignment on a given terrain. The initial vertical alignment as well as convexity-concavity (crest-sag) segments should be determined by the designer. The optimization process has been applied and parameters have had significant effects over solutions.

Major findings of this research as follows:

1. Once vertical alignment optimization has been done, earthwork volume and material costs are calculated accurately in AutoCAD Civil 3D software. The main idea behind using CAD software for earthwork calculations is to create a practical and efficient optimization methodology which simulates real-world scenarios.

2. The optimization method is tested on a real-world terrain and chosen study area was located at the east of Ufa City near Aratskoye village in Chelyabinsk oblast of Russia.

3. The cut-fill cost factors and unit prices used in this paper are hypothetical, however, it depicts the correlation between costs. Consequently, real-world cost factors and unit prices can be applied easily and precisely.

4. The earthwork volumes therefore earthwork costs have been decreased significantly compared to the preliminary design by the application of the optimization procedure.

5. It has been found that developed linear optimization model can be used effectively to minimize earthwork volumes despite its clear disadvantages and shortcomings. 


\section{References}

1. A. B. Goktepe, A. H. Lav, Method for optimizing earthwork considering soil properties in the geometric design of highways. Journal of surveying engineering 130(4), 183-190 (2004)

2. D. Hirpa, W. Hare, Y. Lucet, Y. Pushak, S. Tesfamariam, A bi-objective optimization framework for three-dimensional road alignment design. Transportation Research Part C: Emerging Technologies 65, 61-78 (2016)

3. D. Li, M. Lu, Automated generation of work breakdown structure and project network model for earthworks project planning: a flow network-based optimization approach Journal of Construction Engineering and Management 143(1), 04016086 (2016)

4. D. Monnet, W. Hare, Y. Lucet, Fast feasibility check of the multi-material vertical alignment problem in road design. Computational Optimization and Applications, 1-22 (2019)

5. M. K. Jha, Criteria-Based Support System for Selecting Highway Alignments, Journal of Transportation. Engineering, ASCE, 129(1), 33-41 (2003)

6. S. M. Easa, State-of-the-Art of Three-Dimensional Highway Geometric Design. Canadian Journal of Civil Engineering 25, 500-511 (1997)

7. T. F. Fwa, W. T. Chan, Y. P. Sim, Optimal Vertical Alignment Analysis for Highway Design. Journal of Transportation Engineering 5(128), 395-402 (2002)

8. T. F. Fwa, Highway Vertical Alignment Analysis by Dynamic Programming Transportation Research Record 1239, pp. 1-12 (1989)

9. A. B. Goktepe, S. Altun, P. Ahmedzade, Optimization of vertical alignment of highways utilizing discrete dynamic programming and weighted ground line. Turkish Journal of Engineering and Environmental Sciences 2(33), 105-116 (2009)

10. A. B. Goktepe, A. H. Lav, S. Altun, Dynamic Optimization Algorithm for Vertical Alignment of Highways. Mathematical and Computational Applications 3(10), 341-350 (2005)

11. W. Li, H. Pu, H. Zhao, W. Liu, Approach for Optimizing 3D Highway Alignments Based on Two-stage Dynamic Programming. JSW 8(11), 2967-2973 (2013)

12. C. J. Goh, E. P. Chew, T. F. Fwa, Discrete and continuous models for computation of optimal vertical highway alignment. Transportation Research, Part B 6(22), 399-409 (1988)

13. A. B. Goktepe, A. H. Lav, S. Altun, Method for Optimal Vertical Alignment of Highways. Proceedings of the Institution of Civil Engineers 162, 177-188 (2009)

14. W. L. Hare, S. Hossain, Y. Lucet, F. Rahman, Models and Strategies for Efficiently Determining an Optimal Vertical Alignment of Roads. Computers \& Operations Research, 44, 161-173 (2014)

15. M. Parente, A. G. Correia, P. Cortez, A. Novel, Integrated Optimization System for Earthwork Tasks. Transportation Research Procedia 14, 3601-3610 (2016)

16. A. S. A. Al-Sobky, An Optimization Approach for Highway Vertical Alignment Using the Earthwork Balance Condition. World Applied Sciences Journal 7(29), 884-891 (2014)

17. E. P.Chew, C. J. Goh, T. F. Fwa, Simultaneous optimization of horizontal and vertical alignments for highways. Transportation Research, Part B 5(23B), 315-329 (1989)

18. S. M. Easa, Selection of Roadway Grades that Minimize Earthwork Cost Using Linear Programming. Transportation Research, Part A 2(22), 121-136 (1988) 
19. W. Hare, V. Koch, Y Lucet, Models and algorithms to improve earthwork operations in road design using mixed integer linear programming. European Journal of Operational Research 215, 470-480 (2011)

20. W. L. Hare, Y. Lucet, F. Rahman, A mixed-integer linear programming model to optimize the vertical alignment considering blocks and side-slopes in road construction. European journal of operational research, 241(3), 631-641 (2015) 